\title{
Observation of 2D Conduction in Ultrathin Germanium Arsenide Field-Effect Transistors
}

Grillo, Alessandro; Di Bartolomeo, Antonio; Urban, Francesca; Passacantando, Maurizio; Caridad, Jose M.; Sun, Jianbo; Camilli, Luca

\author{
Published in: \\ ACS Applied Materials and Interfaces
}

Link to article, DOI:

10.1021/acsami.0c00348

Publication date:

2020

Document Version

Publisher's PDF, also known as Version of record

Link back to DTU Orbit

Citation (APA):

Grillo, A., Di Bartolomeo, A., Urban, F., Passacantando, M., Caridad, J. M., Sun, J., \& Camilli, L. (2020).

Observation of 2D Conduction in Ultrathin Germanium Arsenide Field-Effect Transistors. ACS Applied Materials and Interfaces, 12(11), 12998-13004. https://doi.org/10.1021/acsami.0c00348

\section{General rights}

Copyright and moral rights for the publications made accessible in the public portal are retained by the authors and/or other copyright owners and it is a condition of accessing publications that users recognise and abide by the legal requirements associated with these rights.

- Users may download and print one copy of any publication from the public portal for the purpose of private study or research.

- You may not further distribute the material or use it for any profit-making activity or commercial gain

- You may freely distribute the URL identifying the publication in the public portal 


\title{
Observation of 2D Conduction in Ultrathin Germanium Arsenide Field-Effect Transistors
}

\author{
Alessandro Grillo, Antonio Di Bartolomeo,* Francesca Urban, Maurizio Passacantando, Jose M. Caridad, \\ Jianbo Sun, and Luca Camilli*
}

Cite This: ACS Appl. Mater. Interfaces 2020, 12, 12998-13004

Read Online

ACCESS I

Џll Metrics \& More

回国 Article Recommendations

ABSTRACT: We report the fabrication and electrical characterization of germanium arsenide (GeAs) field-effect transistors with ultrathin channels. The electrical transport is investigated in the 20-280 $\mathrm{K}$ temperature range, revealing that the p-type electrical conductivity and the field-effect mobility are growing functions of temperature. An unexpected peak is observed in the temperature dependence of the carrier density per area at $\sim 75 \mathrm{~K}$. Such a feature is explained considering that the increased carrier concentration at higher temperatures and the vertical band bending combined with the gate field lead to the formation of a two-dimensional (2D) conducting channel, limited to few interfacial GeAs layers, which dominates the channel conductance. The conductivity follows the variable-range hopping model at low temperatures and becomes the band-type at higher temperatures when the $2 \mathrm{D}$ channel is formed. The formation of the $2 \mathrm{D}$ channel is validated through a numerical simulation that shows excellent agreement with the experimental data.

KEYWORDS: germanium arsenide, 2D conduction, temperature-dependent conduction, field-effect transistors, carrier density, mobility, variable-range hopping

\section{INTRODUCTION}

Since the isolation of graphene, ${ }^{1}$ there has been growing interest in the research of new two-dimensional (2D) materials for the realization of next-generation electronic devices., ${ }^{2,3}$ Several transition metal dichalcogenides (TMDs) have promptly emerged as possible substitutes or complements to graphene ${ }^{4}$ and have already been investigated and proposed for several applications. ${ }^{5-13}$ More recently, the search for new types of $2 \mathrm{D}$ materials has led to the investigation of binary compounds of IV and V groups. Indeed, theoretical calculations have indicated that $\mathrm{MX}$ compounds, with $\mathrm{M}=$ $\mathrm{Si}, \mathrm{Ge}, \mathrm{Sn}$, or $\mathrm{Pb}$ and $\mathrm{X}=\mathrm{P}, \mathrm{As}, \mathrm{Sb}$, or $\mathrm{Bi}$, might have crystalline layered structures with orthorhombic $\left(\mathrm{Cmc}_{1}\right.$ for $\mathrm{SiP}$ and $\mathrm{Pbam}$ for $\mathrm{SiP}_{2}$ and $\mathrm{GeAs}_{2}$ ) or monoclinic $(\mathrm{C} 2 / \mathrm{m}$ for $\mathrm{GeP}, \mathrm{GeAs}$, and SiAs) symmetries. ${ }^{14-17}$ Several studies have investigated the band gap, ${ }^{18}$ lattice structure, ${ }^{14}$ thermoelectric performances, ${ }^{19}$ and the Hall mobility ${ }^{20}$ of such materials as bulk crystals, but there have been only a few reports on their electrical properties in the form of ultrathin or monolayer flakes. $^{21,22}$

Among the IV-V compounds, GeAs has attracted attention for possible optoelectronic applications and for its high inplane anisotropy. ${ }^{21,22}$ GeAs bulk crystalizes in a layered structure, in the space group $C 2 / m$, in which each $\mathrm{Ge}$ atom is bonded to one $\mathrm{Ge}$ atom and three As atoms forming distorted As 6 @Ge 2 octahedra. ${ }^{23}$ GeAs layers, terminated by As atoms, are kept together by weak van der Waals forces and show two different types of $\mathrm{Ge}-\mathrm{Ge}$ bonds. One type is parallel and the other one is perpendicular to the layer plane, highlighting the anisotropic nature of the GeAs crystal structure (see Figure 1a). Similar to TMDs, the GeAs band gap changes according to the number of layers. Numerical calculations and optical band gap measurements indicate that germanium arsenide indirect band gap ranges from $\sim 0.57$ to $0.65 \mathrm{eV}$ for the bulk ${ }^{19,20}$ to $1.6-2.1 \mathrm{eV}$ for the monolayer. ${ }^{24,25}$

In this work, we report the electrical characterization of GeAs back-gate field-effect transistors fabricated using exfoliated flakes of $12 \mathrm{~nm}$ thickness. We measure the output and transfer characteristics in a four-probe configuration, highlighting the p-type nature of the GeAs flakes in a vacuum. We investigate the temperature behavior of the transistors in a

Received: January 7, 2020

Accepted: February 26, 2020

Published: February 26, 2020 

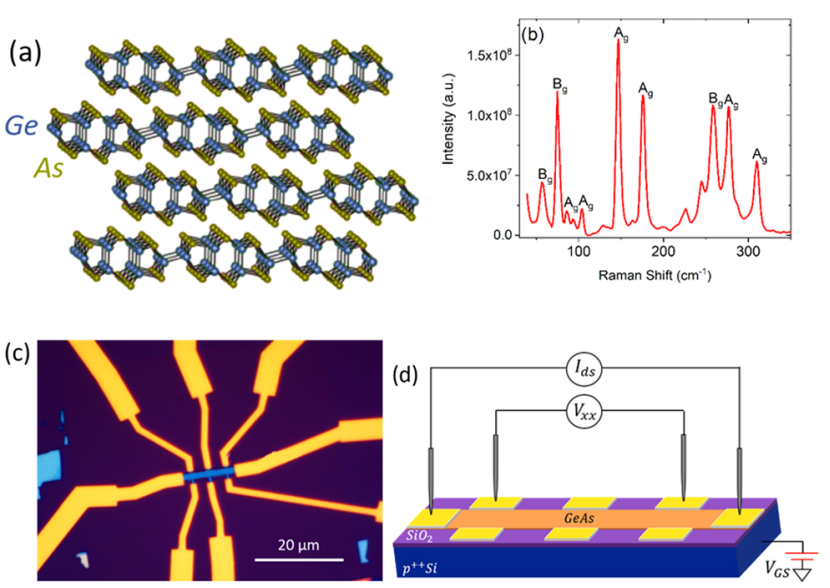

Figure 1. (a) Atom arrangement in GeAs layers. (b) Raman spectrum of a GeAs flake. (c) Optical image of the Hall bar device with $\mathrm{Ni} / \mathrm{Au}$ electrodes. (d) Four-probe measurement setup.

range from 20 to $280 \mathrm{~K}$, estimating figures of merit such as channel resistivity and field-effect mobility. The free carrier density per area, studied as a function of temperature, shows an unexpected broad peak at around $75 \mathrm{~K}$. To explain this anomaly, we propose a model based on the modulation of the channel thickness with temperature. At low temperatures, the intrinsic carrier concentration in the channel is limited and the electric field of the gate controls the whole thickness of the GeAs flake. At higher temperatures, the increased carrier injection from the contacts and ionization of defects enable the formation of a $2 \mathrm{D}$ highly conductive channel close to the dielectric interface, which screens the gate field and confines it to the first few layers of the material. Such a model is corroborated by the estimation of an $\sim 0.4 \mathrm{~nm}$ Debye screening length at room temperature. We compare the numerical simulation of the model with the experimental data obtaining an excellent agreement. To account for the temperature behavior of the resistivity, we use the variable-range hopping (VRH) model at lower temperatures and the thermally activated band-conduction mechanism at higher temperatures. We find that at lower temperatures, the GeAs flake behaves like a three-dimensional system in which the main conduction mechanism is hopping among intragap states, while at higher temperatures, after the formation of the $2 \mathrm{D}$ channel, the conduction is dominated by thermal activation of carriers (holes) to the valence band, which leads to the band-type conduction regime.

\section{MATERIALS AND METHODS}

Ultrathin GeAs flakes were exfoliated from bulk GeAs single crystals using a standard mechanical exfoliation method by adhesive tape. The flakes were transferred onto degenerately doped p-type silicon substrates, covered by $300 \mathrm{~nm}$-thick $\mathrm{SiO}_{2}$, on which they were located through optical microscopy. The Raman spectrum of a flake (Figure $1 \mathrm{~b}$ ) was measured under an excitation line of $455 \mathrm{~nm}$ by a Thermo Scientific DXR microscope and, in agreement with other studies, ${ }^{26}$ it displays six Raman $A_{g}$ modes at 94, 105, 147, 174, 276, and $308 \mathrm{~cm}^{-1}$ and three Raman $\mathrm{B}_{\mathrm{g}}$ modes at 58,76 , and $257 \mathrm{~cm}^{-1}$. By means of an atomic force microscope (AFM), we selected a flake of $\sim 12 \mathrm{~nm}$ thickness, corresponding to about 20 layers, on which we performed standard electron-beam lithography, realizing a typical Hall bar structure, intentionally oriented along one of the crystallographic axes of the material. We then used electron-beam evaporation to deposit $5 \mathrm{~nm} \mathrm{Ni} / 40 \mathrm{~nm} \mathrm{Au}$ as electrodes (Figure 1c). A back-gate contact was formed covering the scratched area of the Si substrate with silver paste.

Electrical measurements were carried out in an Oxford Instrument Teslatron PT cryostat electrically connected with a semiconductor parameter analyzer to perform the standard four-probe characterization of the devices (Figure 1d). All of the measurements were carried out at a pressure of $\sim 10^{-5}$ mbar within the temperature range from 20 to $280 \mathrm{~K}$.

\section{RESULTS AND DISCUSSION}

We show in Figure 2 the electrical characterization of the GeAs transistor at room temperature. The output characteristics, i.e., the drain-source current $\left(I_{\mathrm{ds}}\right)$ as a function of the voltage drop between two inner contacts $\left(V_{\mathrm{xx}}\right)$ with the gate-source voltage $\left(V_{\mathrm{gs}}\right)$ as a control parameter, exhibit linear behavior. The
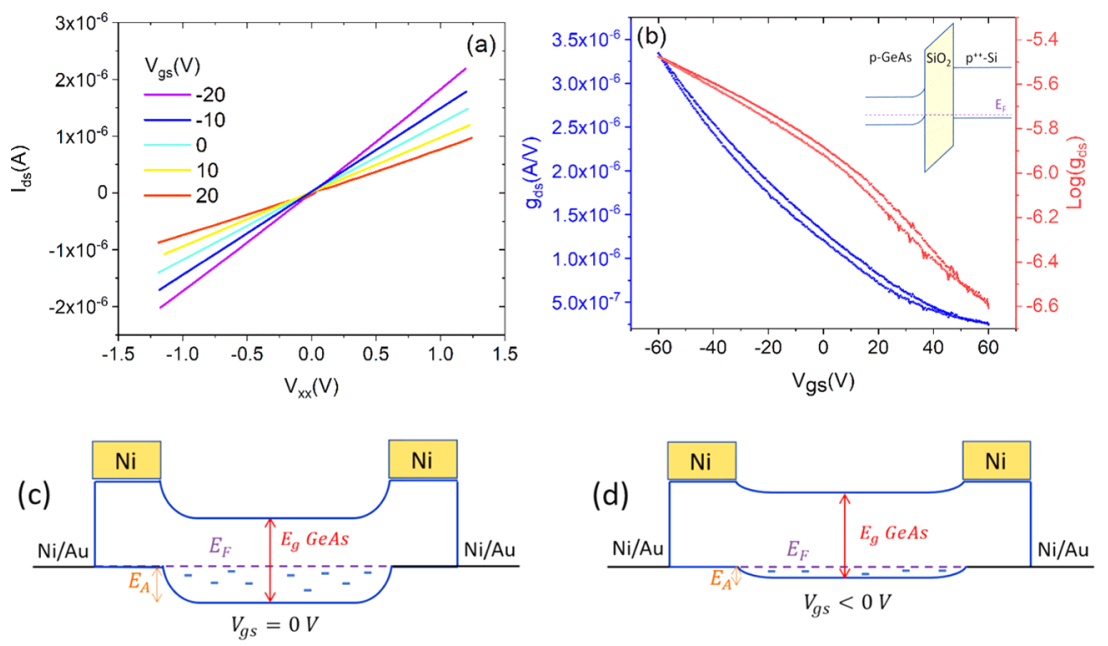

Figure 2. (a) Output characteristic recorded in the four-probe configuration with the $V_{\mathrm{gs}}$ bias being between -20 and $20 \mathrm{~V}$. (b) Transfer characteristic recorded in the four-probe configuration showed both on linear and logarithmic scales. The inset shows the band diagram in the vertical direction at zero bias clarifying that the device is a normally-on transistor. (c, d) Band diagram along the channel (horizontal) direction, showing alignment between $\mathrm{Ni} / \mathrm{Au}$ contacts and GeAs at $V_{\mathrm{gs}}=0$ and $V_{\mathrm{gs}}<0$, respectively. The small blue dashes represent the (mostly empty) trap states for holes. The contact region is more doped than the channel (not on scale), thus giving rise to an energy barrier $E_{\mathrm{A}}$, which is modulated by the gate. 

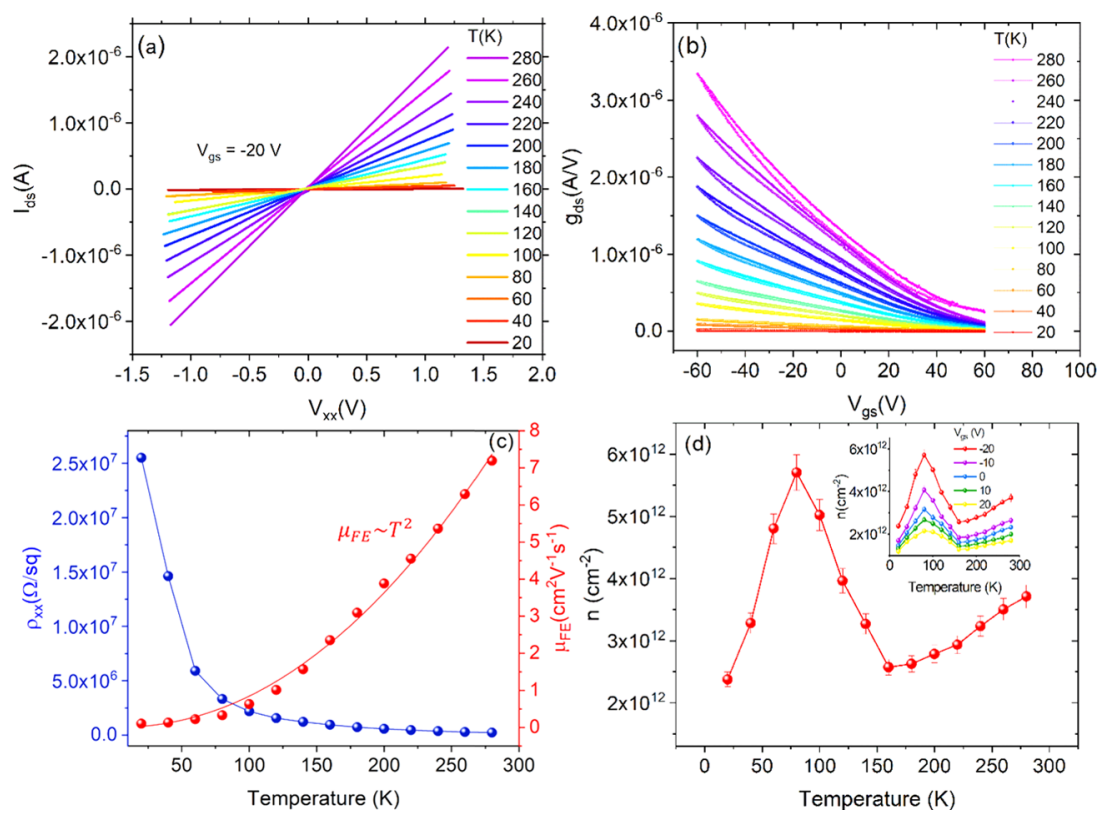

Figure 3. (a) $I_{\mathrm{ds}}$ versus $V_{\mathrm{xx}}$ curves at different temperatures. (b) Transfer characteristics recorded for a loop of the voltage bias at different temperatures. (c) Channel resistivity (blue dots) and field-effect mobility (red dots) as a function of temperature. The superposed red line is the quadratic fit. (d) Carrier density $n$ versus temperature at $V_{\mathrm{GS}}=-20 \mathrm{~V}$. The inset shows $n$ as a function of temperature at several gate biases. The uncertainties are obtained from the standard error propagation.

application of the gate voltage affects the overall conductance of the sample without modifying the $I_{\mathrm{ds}}-V_{\mathrm{xx}}$ linearity as expected for four-probe measurements that exclude the effect of the contact resistance. ${ }^{27-29}$ The transfer characteristic, i.e., the $g_{\mathrm{ds}}-V_{\mathrm{gs}}$ curve $\left(g_{\mathrm{ds}}=I_{\mathrm{ds}} / V_{\mathrm{xx}}\right.$ is the channel conductance) measured over a loop of the gate voltage is reported in Figure $2 \mathrm{~b}$. It shows a typical p-type behavior with a modulation that is less than 1 order of magnitude. We did not reach the off-state of the transistor in the applied voltage range, which was limited to avoid the breaking of the device gate dielectric. The p-type behavior is favored by the low work function ${ }^{15}$ of GeAs ( $\sim 4$ $\mathrm{eV}$ ) and the higher work function of the degenerate $\mathrm{p}$-Si gate $(>5.12 \mathrm{eV})$ that cause the vertical band alignment shown in the inset of Figure 2b. Furthermore, the Ni Fermi level (work function of $5.15 \mathrm{eV}$ ) tends to align below the valence band of GeAs, resulting in ohmic contacts. Furthermore, intrinsic defects such as $\mathrm{Ge}$ vacancies, the interaction with the $\mathrm{SiO}_{2}$ gate dielectric, and the oxidation of the topmost layers of the flake would act as p-type dopants in the material and provide intragap states. ${ }^{14,15}$ Hole injection from the metal leads makes the area below and near the contacts more doped than the rest of the long channel, thereby giving rise to an energy barrier $E_{\mathrm{A}}$ which can be modulated by the gate (see Figure $2 \mathrm{c}, \mathrm{d}$ ). ${ }^{30}$

Differently from few-layer TMD-based transistors, ${ }^{31-35}$ the transfer characteristic exhibits negligible hysteresis indicating limited charge trapping during a gate voltage loop.

The temperature dependence of the electric behavior of the device is investigated in Figure 3. We measured the output and transfer characteristics every $20 \mathrm{~K}$ in the $20-280 \mathrm{~K}$ temperature range (Figure $3 a, b)$. We found a decreasing current and conductance with lowering temperature without any other apparent change in the behavior of the device. From the linear fit of the $I_{\mathrm{ds}}-V_{\mathrm{xx}}$ curves, recorded at $V_{\mathrm{gs}}=-20 \mathrm{~V}$, we estimated the resistivity $\rho$ of the sample as a function of the temperature ( $\rho=\frac{R W}{L}$, where $R$ is the resistance evaluated from the fit, and $L=6$ and $W=2.7 \mu \mathrm{m}$ are the channel length and width, respectively). Figure $3 \mathrm{c}$ shows a decreasing $\rho$ for increasing temperature, typical of a semiconducting material.

Using the transfer characteristics, considering their nonlinear behavior, we expressed the field-effect transistor (FET) channel conductance as

$$
g_{\mathrm{ds}}=\frac{I_{\mathrm{ds}}}{V_{\mathrm{xx}}}=\frac{W}{L} \mu_{\mathrm{FE}} C_{\mathrm{ox}}\left|V_{\mathrm{gs}}-V_{\mathrm{th}}\right|^{\alpha}
$$

where $\mu_{\text {FE }}$ is the field-effect mobility; $C_{\mathrm{ox}}=\frac{\epsilon_{0} \cdot \epsilon_{\mathrm{SiO}_{2}}}{t_{\mathrm{SiO}_{2}}}=1.15 \times 10^{-8}\left(\mathrm{~F} / \mathrm{cm}^{2}\right)$ is the capacitance per unit area of the gate dielectric, where $\epsilon_{0}=8.85 \times 10^{-14} \mathrm{~F} / \mathrm{cm}^{2}$, $\epsilon_{\mathrm{SiO}_{2}}=3.9$, and $t_{\mathrm{SiO}_{2}}=300 \mathrm{~nm}$ are the vacuum permittivity, the $\mathrm{SiO}_{2}$ relative permittivity, and thickness, respectively; $V_{\text {th }}$ is the threshold voltage; and $\alpha \geq 1$ is a dimensionless parameter that accounts for a possible $V_{\mathrm{gs}}$-dependence of mobility. ${ }^{31}$ According to eq 1 , when the $I_{\mathrm{ds}}-V_{\mathrm{gs}}$ curve is linear and $\alpha=$ 1 , the mobility can be obtained as

$$
\mu_{\mathrm{FE}}=\frac{L}{W} \frac{1}{C_{\mathrm{ox}}} \frac{\mathrm{d} g_{\mathrm{ds}}}{\mathrm{d} V_{\mathrm{gs}}}
$$

To have a fair comparison with the values of $\rho$ obtained from $g_{\mathrm{ds}}-V_{\mathrm{xx}}$ curves at $V_{\mathrm{gs}}=-20 \mathrm{~V}$, we performed the linear fit of the transfer characteristics in a small interval around $V_{\mathrm{gs}}=$ $-20 \mathrm{~V}$. Figure $3 \mathrm{c}$ shows the mobility $\mu_{\mathrm{FE}}$ as a function of temperature $T$. The quadratic dependence, $\mu_{\mathrm{FE}} \sim T^{2}$, points toward a mobility dominated by Coulomb scattering due to ionized impurities. ${ }^{36}$ Indeed, it is well known that the mobility is affected by two competitive mechanisms, i.e., the ionized impurity scattering and the phonon scattering. ${ }^{36}$ The first mechanism dominates at lower temperatures and yields increasing mobility with increasing temperature, while phonon scattering becomes the prevailing mechanism at higher temperatures and causes decreasing mobility. As already mentioned, charged impurities leading to Coulomb scattering 
(a) - Carriers: low temperature

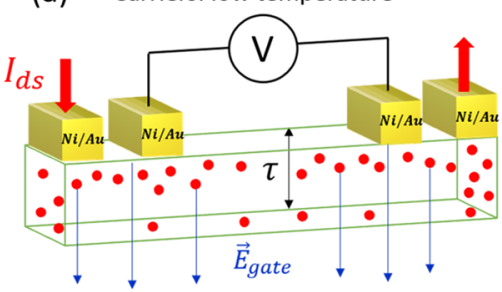

(b) - Carriers: high temperature
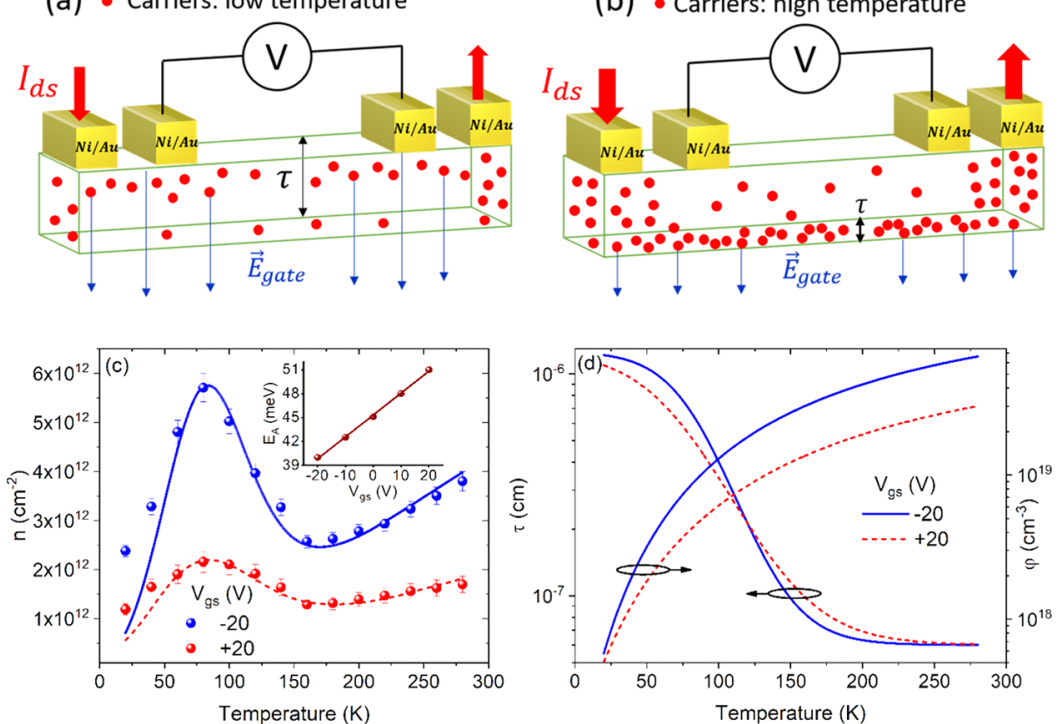

Figure 4. (a) Schematic of the GeAs flake at low temperatures with low carrier density and the electric field of the gate at $V_{\mathrm{GS}}<0 \mathrm{~V}$ (blue arrows) controlling the entire thickness. (b) Schematic of the GeAs flake at high temperatures with the formation of a highly conductive channel confined to a thin interfacial layer, which screens the electric field of the gate (blue arrows). The drawings are not on the scale. (c) Numerical simulation of the $2 \mathrm{D}$ carrier density as a function of temperature for $V_{\mathrm{gs}}=\mp 20 \mathrm{~V}$ (fitting lines superposed the experimental data). The inset shows $E_{\mathrm{a}}$ as a function of the applied gate potential. (d) Effective thickness of the channel (blue lines) and three-dimensional carrier density (red lines) as a function of temperature for $V_{\mathrm{gs}}=\mp 20 \mathrm{~V}$.

and increasing $\mu_{\mathrm{FE}}$ are due to the intrinsic defects of GeAs, the $\mathrm{SiO}_{2}$ interface states, and the oxidation of the topmost layers of the flake.

Having computed $\mu_{\mathrm{FE}}(T)$ and $\rho(T)$ at each of the considered temperatures, we estimated the carrier density per unit area $n\left(\right.$ in $\left.\mathrm{cm}^{-2}\right)$ as a function of temperature from the relation

$$
n(T)=\frac{1}{q \rho(T) \mu_{\mathrm{FE}}(T)}
$$

where $q$ is the electron charge. Figure $3 \mathrm{~d}$ shows that $n(T)$ exhibits an unexpected and pronounced peak at $T \sim 75 \mathrm{~K}$, followed by a smoother increase for $T>150 \mathrm{~K}$. In a doped three-dimensional semiconductor, the carrier density would show a different temperature behavior. ${ }^{36}$ Specifically, an initial rapid increase due to the ionization of dopant atoms by thermal energy (freeze-out region, up to $\sim 150 \mathrm{~K}$ for mediumdoped $\mathrm{Si}$ ) would be followed by a plateau over a wide temperature range when complete ionization is reached (extrinsic region, $\sim 150$ to $500 \mathrm{~K}$ for medium-doped $\mathrm{Si}$ ) and would finally evolve in an exponential increase at higher temperatures due to the intrinsic generation of electron-hole pairs (intrinsic region).

To explain the reported anomalous behavior, we propose a model that considers a temperature-dependent thickness of the channel in which most of the conduction occurs. The 2D carrier density per unit area can be expressed as $n(T)=$ $\varphi(T) \tau(T)$, where $\varphi$ is the carrier density per unit volume in $\mathrm{cm}^{-3}$ and $\tau$ is the thickness of the conducting channel. The current in the GeAs flake is due to the carriers injected from the $\mathrm{Ni}$ contacts that overcome the $E_{\mathrm{A}}$ barrier (Figure 2c,d). Therefore, $\varphi(T)$ in the region between the inner contacts (Figure $4 \mathrm{a}, \mathrm{b}$ ) can be expressed as ${ }^{37}$

$$
\varphi(T)=A \exp \left[-\frac{E_{\mathrm{A}}}{k T}\right]
$$

where $k$ is the Boltzmann constant, $E_{\mathrm{A}}$ is the barrier between contact and channel regions, and $A$ is a proportionality constant that can be considered as a fitting parameter of the model. At low temperature $(<80 \mathrm{~K})$, there is a limited amount of carriers as well as high intralayer resistance that suppresses the vertical transport with respect to the in-plane one. ${ }^{30,38}$ In this regime, conduction occurs mainly through the layers in closer contact with the metal leads (especially the topmost ones) and the gate electric field controls the entire flake thickness (Figure 4a). With increasing temperature, defect ionization and injection from the contacts increase. The newly available carriers, pushed by the applied gate voltage and the favorable vertical band bending (see the inset of Figure $2 b$ ) toward the interface with the gate dielectric, form a $2 \mathrm{D}$ channel, which becomes more and more conductive with increasing temperature. This channel now screens the gate field, and any variation of the gate voltage affects mainly the bottommost layers of the flake. In this regime, the conduction is dominated by few atomic GeAs layers closer to the gate, i.e., the effective conducting thickness $\tau(T)$ controlled by the gate is reduced (Figure $4 \mathrm{~b}$ ).

Such a model is confirmed by the measurement of $n(T)$ at various gate biases, shown in the inset of Figure $3 \mathrm{~d}$. Indeed, the $2 \mathrm{D}$ channel at the interfacial region between the gate dielectric and the flake is hampered at positive gate voltages by the opposing gate field. Going to positive gate voltages, the $2 \mathrm{D}$ conductive channel is gradually suppressed and so is the carrier density $n$ and its peak at $\sim 75 \mathrm{~K}$.

According to the model, with an appropriate choice of $\varphi(T)$ and $\tau(T)$, we computed $n(T)$ and obtained an excellent agreement with the experimental data, as shown in Figure 4c, where we display $n(T)$ at $V_{\mathrm{gs}}=-20 \mathrm{~V}$ (continuous blue line) as well as at $V_{\mathrm{gs}}=20 \mathrm{~V}$ (dashed red line). The slight deviation below $50 \mathrm{~K}$ could be caused by additional conduction mechanisms occurring at low temperature (such as hopping, see later). The carrier density per volume $\varphi(T)$, given by eq 4 , 

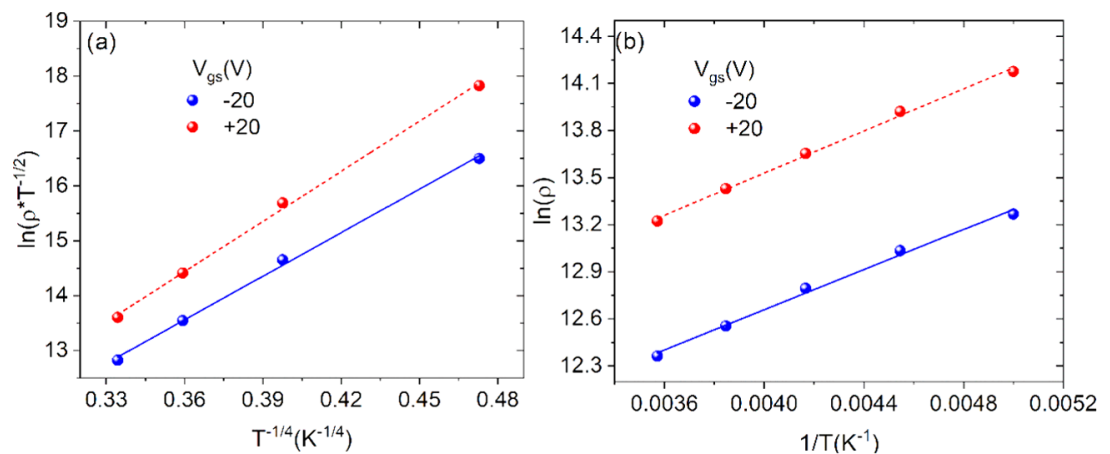

Figure 5. (a) Experimental data and linear fit of $\ln \left(\rho T^{-1 / 2}\right)$ at $V_{\mathrm{gs}}=\mp 20 \mathrm{~V}$ as a function of $T^{-1 / 4}$ at low temperature, indicating the threedimensional nature of the investigated system. (b) Experimental data and linear fit of $\ln (\rho)$ at $V_{\mathrm{gs}}=\mp 20 \mathrm{~V}$ as a function of $T^{-1}$ at high temperature, indicating the band conduction regime of the investigated system.

is shown by the red lines of Figure $4 \mathrm{~d}$, with $E_{\mathrm{A}}$ being obtained as a fitting parameter and displayed as a function of $V_{\mathrm{gs}}$ in the inset of Figure 4c. For $\tau(T)$, we used a steplike function, $\tau(T)=\frac{A}{1+\exp (B T)}+C$, represented by the blue curves shown in Figure $4 d$, in which $A$ is the initial thickness of the whole flake, i.e., $\sim 12 \mathrm{~nm}, C$ is the thickness of a single GeAs layer $(0.6 \mathrm{~nm}),{ }^{38}$ and $B$ is a fitting parameter used to simulate a smooth transition in the $70-150 \mathrm{~K}$ range.

The model is further corroborated by the estimation of the Debye screening length $L_{\mathrm{D}}=\sqrt{\frac{\epsilon \epsilon_{0} k T}{q^{2} \varphi}}$, which gives the length over which the electric field strength drops by a factor $1 / e$ (here $\epsilon=8$ is the dielectric constant at room temperature of the material $\left.{ }^{39}\right)$. From our data, we estimate $L_{\mathrm{D}} \sim 0.4 \mathrm{~nm}$ at room temperature, confirming that the gate electric field is substantially screened in the layer closer to the gate.

Consistent with our model, we remark that the formation of a conductive channel near the bottom substrate, screening the gate field, and the presence of an energy barrier caused by the inhomogeneous carrier distribution along the channel thickness have been considered to explain the negative transconductance in thick TMD-based back-gated field-effect transistors. ${ }^{30}$ Furthermore, the appearance of two separate $2 \mathrm{D}$ conductive channels, close to the respective gates, due to $\mathrm{S}$ vacancy ionization and gate-field screening, has been recently reported in dual-gated thick $\mathrm{MoS}_{2}$ transistors at room temperature. According to such a study, when the temperature is lowered below $80 \mathrm{~K}$, the two separate conductive channels merge into a single one and the top conductive channel governs the transistor behavior. ${ }^{38}$

We also investigated the resistivity behavior $\rho$ as a function of $T$. The best fitting of the experimental data at low temperature is obtained using the variable-range hopping (VRH) conduction. We notice that VRH is usually observed in amorphous solids as well as in defective systems at low temperature and has been reported in several two-dimensional materials. ${ }^{40,41}$ According to VRH theory, the relation between $\rho$ and $T$ can be expressed as ${ }^{42}$

$$
\rho(T)=\rho_{0} \exp \left(\frac{T_{0}}{T}\right)^{1 / n+1}
$$

where $\rho_{0}$ depends on the square root of $T, T_{0}$ is a constant, and $\mathrm{n}$ indicates the dimensionality of the system. ${ }^{43}$ Figure 5 a shows $\ln \left(\rho T^{-1 / 2}\right)$ as a function of $T^{-1 / 4}$ in the range $20-80 \mathrm{~K}$. The linearity demonstrates that the conduction is bulk type for $T<$
$80 \mathrm{~K}$, i.e., it occurs through the entire flake constituting $\sim 20$ atomic layers, as assumed in the proposed model. For $T>180$ $\mathrm{K}, \rho$ shows an exponential increase with the inverse of temperature (Figure $5 \mathrm{~b}$ ). This indicates that the thermal excitation of carriers (holes) to the valence band leads to a band-conduction regime, $\rho \propto \exp \left(\frac{E_{\mathrm{A}}}{k T}\right)$, as expected after the formation of a highly conductive $2 \mathrm{D}$ channel in the GeAs transistor.

\section{CONCLUSIONS}

We fabricated back-gate field-effect transistors with ultrathin GeAs films and investigated their electrical properties over a wide temperature range from 20 to $280 \mathrm{~K}$. We found p-type behavior with temperature upon increasing conductivity. We observed that the carrier density in GeAs flakes depends on temperature with a pronounced and broad peak at around 75 $\mathrm{K}$. We proposed a model based on temperature-dependent channel thickness to explain such an anomaly. We showed that the electrical conduction of the GeAs channel can be explained by variable-range hopping at lower temperatures but it becomes the band-type at higher temperatures when a highly conducting $2 \mathrm{D}$ channel is formed. The proposed model, validated by numerical simulation, shows an excellent agreement with the experimental data.

This study provides a new understanding of the intrinsic properties of few-layer GeAs as the channel of field-effect transistors, providing evidence of the formation of a $2 \mathrm{D}$ channel limited to a single atomic layer and could be applied to other ultrathin layered materials.

\section{AUTHOR INFORMATION}

\section{Corresponding Authors}

Antonio Di Bartolomeo - Physics Department "E. R. Caianiello", University of Salerno, Fisciano 84084, Italy; CNRSPIN Salerno, Fisciano 84084, Italy; 이이.org/0000-00023629-726X; Email: adibartolomeo@unisa.it

Luca Camilli - Department of Physics, Technical University of Denmark, 2800 Kgs. Lyngby, Denmark; 이이.org/00000003-2498-0210; Email: lcam@dtu.dk

\section{Authors}

Alessandro Grillo - Physics Department "E. R. Caianiello", University of Salerno, Fisciano 84084, Italy; CNR-SPIN Salerno, Fisciano 84084, Italy; 이이이.org/0000-0002-89099865 
Francesca Urban - Physics Department "E. R. Caianiello", University of Salerno, Fisciano 84084, Italy; CNR-SPIN Salerno, Fisciano 84084, Italy; 이이이.org/0000-0003-21091370

Maurizio Passacantando - Department of Physical and Chemical Science, University of L'Aquila and CNR-SPIN L'Aquila, L'Aquila 67100, Coppito, Italy; (i) orcid.org/00000002-3680-5295

Jose M. Caridad - Department of Physics, Technical University of Denmark, 2800 Kgs. Lyngby, Denmark

Jianbo Sun - Department of Physics, Technical University of Denmark, 2800 Kgs. Lyngby, Denmark; 이이이.org/00000001-9741-8726

Complete contact information is available at: https://pubs.acs.org/10.1021/acsami.0c00348

\section{Notes}

The authors declare no competing financial interest.

\section{ACKNOWLEDGMENTS}

L.C. and J.S. are grateful to Villum Fonden for financial support (Young Investigator Program, Project No. 19130). A.D.B. acknowledges the financial support of MIUR Project Pico \& Pro, ARS01_01061, 2018-2021. L.C. acknowledges support from the Italian Ministry of Education, University and Research (MIUR) via "Programma per Giovani Ricercatori Rita Levi Montalcini 2017”.

\section{REFERENCES}

(1) Novoselov, K. S.; et al. Electric Field Effect in Atomically Thin Carbon Films. Science 2004, 306, 666-669.

(2) Fiori, G.; Bonaccorso, F.; Iannaccone, G.; Palacios, T.; Neumaier, D.; Seabaugh, A.; Banerjee, S. K.; Colombo, L. Electronics Based on Two-Dimensional Materials. Nat. Nanotechnol. 2014, 9, 768-779.

(3) Lemme, M. C.; Li, L.-J.; Palacios, T.; Schwierz, F. TwoDimensional Materials for Electronic Applications. MRS Bull. 2014, 39, 711-718.

(4) Manzeli, S.; Ovchinnikov, D.; Pasquier, D.; Yazyev, O. V.; Kis, A. 2D Transition Metal Dichalcogenides. Nat. Rev. Mater. 2017, 2, No. 17033.

(5) Jiao, Y.; Hafez, A. M.; Cao, D.; Mukhopadhyay, A.; Ma, Y.; Zhu, H. Metallic $\mathrm{MoS}_{2}$ for High Performance Energy Storage and Energy Conversion. Small 2018, 14, No. 1800640.

(6) Di Bartolomeo, A.; Urban, F.; Passacantando, M.; McEvoy, N.; Peters, L.; Iemmo, L.; Luongo, G.; Romeo, F.; Giubileo, F. A WSe2 Vertical Field Emission Transistor. Nanoscale 2019, 11, 1538-1548.

(7) Urban, F.; Martucciello, N.; Peters, L.; McEvoy, N.; Di Bartolomeo, A. Environmental Effects on the Electrical Characteristics of Back-Gated WSe2 Field-Effect Transistors. Nanomaterials 2018, 8, 901.

(8) Seo, J.-W. T.; Zhu, J.; Sangwan, V. K.; Secor, E. B.; Wallace, S. G.; Hersam, M. C. Fully Inkjet-Printed, Mechanically Flexible $\mathrm{MoS}_{2}$ Nanosheet Photodetectors. ACS Appl. Mater. Interfaces 2019, 11, 5675-5681.

(9) Di Bartolomeo, A.; Genovese, L.; Foller, T.; Giubileo, F.; Luongo, G.; Croin, L.; Liang, S.-J.; Ang, L. K.; Schleberger, M. Electrical Transport and Persistent Photoconductivity in Monolayer $\mathrm{MoS}_{2}$ Phototransistors. Nanotechnology 2017, 28, No. 214002.

(10) Patel, A. B.; Machhi, H. K.; Chauhan, P.; Narayan, S.; Dixit, V.; Soni, S. S.; Jha, P. K.; Solanki, G. K.; Patel, K. D.; Pathak, V. M. Electrophoretically Deposited $\mathrm{MoSe}_{2} / \mathrm{WSe}_{2}$ Heterojunction from Ultrasonically Exfoliated Nanocrystals for Enhanced Electrochemical Photoresponse. ACS Appl. Mater. Interfaces 2019, 11, 4093-4102.
(11) Grillo, A.; Giubileo, F.; Iemmo, L.; Luongo, G.; Urban, F.; Di Bartolomeo, A. Space Charge Limited Current and Photoconductive Effect in Few-Layer $\mathrm{MoS}_{2}$. J. Phys.: Conf. Ser. 2019, 1226, No. 012013.

(12) Liu, H.; Cui, M.; Dang, C.; Wen, W.; Wang, X.; Xie, L. TwoDimensional $\mathrm{WSe}_{2} /$ Organic Acceptor Hybrid Nonvolatile Memory Devices Based on Interface Charge Trapping. ACS Appl. Mater. Interfaces 2019, 11, 34424-34429.

(13) Iemmo, L.; Urban, F.; Giubileo, F.; Passacantando, M.; Di Bartolomeo, A. Nanotip Contacts for Electric Transport and Field Emission Characterization of Ultrathin MoS2 Flakes. Nanomaterials 2020, 10, No. 106.

(14) Wu, P.; Huang, M. Stability, Bonding, and Electronic Properties of Silicon and Germanium Arsenides: Stability, Bonding, and Electronic Properties of Si and Ge Arsenides. Phys. Status Solidi B 2016, 253, 862-867.

(15) Jung, C. S.; Kim, D.; Cha, S.; Myung, Y.; Shojaei, F.; Abbas, H. G.; Lee, J. A.; Cha, E. H.; Park, J.; Kang, H. S. Two-Dimensional GeAs with a Visible Range Band Gap. J. Mater. Chem. A 2018, 6, 9089-9098.

(16) Cheng, A.-Q.; He, Z.; Zhao, J.; Zeng, H.; Chen, R.-S. Monolayered Silicon and Germanium Monopnictide Semiconductors: Excellent Stability, High Absorbance, and Strain Engineering of Electronic Properties. ACS Appl. Mater. Interfaces 2018, 10, 51335139.

(17) Shojaei, F.; Kang, H. S. Electronic Structures and Li-Diffusion Properties of Group IV-V Layered Materials: Hexagonal Germanium Phosphide and Germanium Arsenide. J. Phys. Chem. C 2016, 120, 23842-23850.

(18) Miao, M.-s.; Botana, J.; Zurek, E.; Hu, T.; Liu, J.; Yang, W. Electron Counting and a Large Family of Two-Dimensional Semiconductors. Chem. Mater. 2016, 28, 1994-1999.

(19) Lee, K.; Kamali, S.; Ericsson, T.; Bellard, M.; Kovnir, K. GeAs: Highly Anisotropic van Der Waals Thermoelectric Material. Chem. Mater. 2016, 28, 2776-2785.

(20) Rau, J. W.; Kannewurf, C. R. Optical Absorption, Reflectivity, and Electrical Conductivity in GeAs and GeAs2. Phys. Rev. B 1971, 3, 2581-2587.

(21) Guo, J.; Liu, Y.; Ma, Y.; Zhu, E.; Lee, S.; Lu, Z.; Zhao, Z.; Xu, C.; Lee, S.-J.; Wu, H.; Kovnir, K.; Huang, Y.; Duan, X. Few-Layer GeAs Field-Effect Transistors and Infrared Photodetectors. Adv. Mater. 2018, 30, No. 1705934.

(22) Yang, S.; Yang, Y.; Wu, M.; Hu, C.; Shen, W.; Gong, Y.; Huang, L.; Jiang, C.; Zhang, Y.; Ajayan, P. M. Highly In-Plane Optical and Electrical Anisotropy of 2D Germanium Arsenide. Adv. Funct. Mater. 2018, 28, No. 1707379.

(23) Blachnik, R.; Irle, E. Das System Gallium-Tellur. J. LessCommon Met. 1985, 113, L1-L3.

(24) Zhou, L.; Guo, Y.; Zhao, J. GeAs and SiAs Monolayers: Novel 2D Semiconductors with Suitable Band Structures. Phys. E 2018, 95, 149-153.

(25) Mortazavi, B.; Shahrokhi, M.; Cuniberti, G.; Zhuang, X. TwoDimensional SiP, SiAs, GeP and GeAs as Promising Candidates for Photocatalytic Applications. Coatings 2019, 9, 522.

(26) Huang, S.; Tatsumi, Y.; Ling, X.; Guo, H.; Wang, Z.; Watson, G.; Puretzky, A. A.; Geohegan, D. B.; Kong, J.; Li, J.; Yang, T.; Saito, R.; Dresselhaus, M. S. In-Plane Optical Anisotropy of Layered Gallium Telluride. ACS Nano 2016, 10, 8964-8972.

(27) Di Bartolomeo, A.; Grillo, A.; Urban, F.; Iemmo, L.; Giubileo, F.; Luongo, G.; Amato, G.; Croin, L.; Sun, L.; Liang, S.-J.; Ang, L. K. Asymmetric Schottky Contacts in Bilayer MoS2 Field Effect Transistors. Advanced Functional Materials 2018, 28, No. 1800657.

(28) Bartolomeo, A. D.; Giubileo, F.; Romeo, F.; Sabatino, P.; Carapella, G.; Iemmo, L.; Schroeder, T.; Lupina, G. Graphene Field Effect Transistors with Niobium Contacts and Asymmetric Transfer Characteristics. Nanotechnology 2015, 26, No. 475202.

(29) Di Bartolomeo, A.; Santandrea, S.; Giubileo, F.; Romeo, F.; Petrosino, M.; Citro, R.; Barbara, P.; Lupina, G.; Schroeder, T.; Rubino, A. Effect of Back-Gate on Contact Resistance and on 
Channel Conductance in Graphene-Based Field-Effect Transistors. Diamond Relat. Mater. 2013, 38, 19-23.

(30) Liu, Y.; Guo, J.; He, Q.; Wu, H.; Cheng, H.-C.; Ding, M.; Shakir, I.; Gambin, V.; Huang, Y.; Duan, X. Vertical Charge Transport and Negative Transconductance in Multilayer Molybdenum Disulfides. Nano Lett. 2017, 17, 5495-5501.

(31) Di Bartolomeo, A.; Genovese, L.; Giubileo, F.; Iemmo, L.; Luongo, G.; Foller, T.; Schleberger, M. Hysteresis in the Transfer Characteristics of $\mathrm{MoS}_{2}$ Transistors. 2D Mater. 2017, 5, No. 015014.

(32) Liu, N.; Baek, J.; Kim, S. M.; Hong, S.; Hong, Y. K.; Kim, Y. S.; Kim, H.-S.; Kim, S.; Park, J. Improving the Stability of HighPerformance Multilayer $\mathrm{MoS}_{2}$ Field-Effect Transistors. ACS Appl. Mater. Interfaces 2017, 9, 42943-42950.

(33) Urban, F.; Giubileo, F.; Grillo, A.; Iemmo, L.; Luongo, G.; Passacantando, M.; Foller, T.; Madauß, L.; Pollmann, E.; Geller, M. P.; Oing, D.; Schleberger, M.; Di Bartolomeo, A. Gas Dependent Hysteresis in $\mathrm{MoS}_{2}$ Field Effect Transistors. 2D Mater. 2019, 6, No. 045049.

(34) Di Bartolomeo, A.; Pelella, A.; Liu, X.; Miao, F.; Passacantando, M.; Giubileo, F.; Grillo, A.; Iemmo, L.; Urban, F.; Liang, S. PressureTunable Ambipolar Conduction and Hysteresis in Thin Palladium Diselenide Field Effect Transistors. Adv. Funct. Mater. 2019, 29, No. 1902483.

(35) Han, K. H.; Kim, G.-S.; Park, J.; Kim, S.-G.; Park, J.-H.; Yu, H.Y. Reduction of Threshold Voltage Hysteresis of $\mathrm{MoS}_{2}$ Transistors with 3-Aminopropyltriethoxysilane Passivation and Its Application for Improved Synaptic Behavior. ACS Appl. Mater. Interfaces 2019, 11, 20949-20955.

(36) Sze, S. M.; Ng, K. K. Physics of Semiconductor Devices, 3rd ed.; Wiley-Interscience: Hoboken, NJ, 2007.

(37) Ang, Y. S.; Yang, H. Y.; Ang, L. K. Universal Scaling Laws in Schottky Heterostructures Based on Two-Dimensional Materials. Phys. Rev. Lett. 2018, 121, No. 056802.

(38) Ji, H.; Ghimire, M. K.; Lee, G.; Yi, H.; Sakong, W.; Gul, H. Z.; Yun, Y.; Jiang, J.; Kim, J.; Joo, M.-K.; Suh, D.; Lim, S. C. Temperature-Dependent Opacity of the Gate Field Inside $\mathrm{MoS}_{2}$ Field-Effect Transistors. ACS Appl. Mater. Interfaces 2019, 11, 29022-29028.

(39) Mead, D. G. Long Wavelength Study of Semiconducting Germanium Arsenide, GeAs. Infrared Phys. 1982, 22, 209-213.

(40) Ghatak, S.; Pal, A. N.; Ghosh, A. Nature of Electronic States in Atomically Thin $\mathrm{MoS}_{2}$ Field-Effect Transistors. ACS Nano 2011, 5, 7707-7712.

(41) Choi, S. J.; Kim, B.-K.; Lee, T.-H.; Kim, Y. H.; Li, Z.; Pop, E.; Kim, J.-J.; Song, J. H.; Bae, M.-H. Electrical and Thermoelectric Transport by Variable Range Hopping in Thin Black Phosphorus Devices. Nano Lett. 2016, 16, 3969-3975.

(42) Mott, N. F. Conduction in Glasses Containing Transition Metal Ions. J. Non-Cryst. Solids 1968, 1, 1-17.

(43) Barreteau, C.; Michon, B.; Besnard, C.; Giannini, E. HighPressure Melt Growth and Transport Properties of SiP, SiAs, GeP, and GeAs 2D Layered Semiconductors. J. Cryst. Growth 2016, 443, 75-80. 